\section{Ductus arteriosus: gene profile for fetal maturation versus postnatal closure}

Advance online publication, 14 June 2017; doi:10.1038/pr.2017.124

To the Editor: In a recent issue of Pediatric Research, Goyal et al. (1) present a cohort of genes reportedly implicated in the antenatal maturation of the ductus arteriosus and subsequent closure of the vessel at birth. Based on their protocol, specifically the timing of collection of neonatal specimens, one should anticipate from this analysis a gene profile with certain features of relevance to the functional sealing of the vessel. However, contrary to this expectation, there is a peculiar downregulation of calmodulin (Calm1) and the absence of a factor, such as RhoB, which has been critically implicated in the cascade of events being initiated by oxygen and ending with the sustained contraction of the ductus (2). An earlier report from our laboratory (3), worth considering in this context, has shown a duct-specific upregulation of Calm1 after birth. In the same study, it has also been demonstrated that RhoB stands out as a prominent element in a hierarchical clustering of genes in the neonatal ductus. A possible explanation for this apparent incongruence may be found in the analytical approach of Goyal et al. (1), where a paired comparison of duct specimens at different developmental stages (i.e., preterm versus term/term versus neonate) might have yielded a final pool of genes in which the expression profile for antenatal maturation outweighs features proper of the functional closure at birth.

Otherwise, our past work (3) aligns itself with that of Goyal et al. (1) in highlighting the role of certain genes in promoting the maturation of the ductus. In particular, we may add support to the proposed involvement of the Wnt-planar cell polarity/Wnt- $\beta$-catenin pathways by showing not only the presence of genes coincident with the listing of Goyal et al. (1), but also the likely contribution of a separate cohort of functionally allied genes (Table 1).

In conclusion, the study of Goyal et al. (1) sheds better light on the maturation process of the ductus, but is seemingly amiss in documenting events that underlie the functional closure of the vessel.
Table 1. Rat ductus arteriosus: birth-related changes in the expression profile of genes belonging to the combined Wnt-PCP and Wnt- $\beta$-catenin pathways

\begin{tabular}{ll}
\hline $\begin{array}{l}\text { Downregulation } \\
\text { Fdz2 }\end{array}$ & Frizzled homologue 2 \\
Ccnd1 & Cyclin D1 \\
Cdh1 & Cadherin 1
\end{tabular}

\begin{tabular}{ll}
$\begin{array}{l}\text { Upregulation } \\
\text { Sfrp }{ }^{\text {a }}\end{array}$ & Secreted frizzled-related protein 4 \\
Cdh5 & Cadherin 5 \\
c-fos & C-fos \\
Junb & Jun B protoncogene \\
Jund & Jun D protoncogene \\
Rhob & Ras homolog family member B \\
Rock2 & Rho-associated coiled-coil forming kinase 2 \\
\hline
\end{tabular}

PCP, planar cell polarity.

Data from our study (3), with entries in bold being common to the study of Goyal et al. (1). Note that the two studies shared, in the whole, the preferential expression of 11 genes.

aListed originally as "unknown" (3) and with a direction of change opposite to that given by Goyal et al. (1).

Disclosure: The authors declare no conflict of interest.

\section{Flavio Coceani ${ }^{1}$, Francesca Scebba ${ }^{1}$ and Debora Angeloni ${ }^{1}$ \\ ${ }^{1}$ Institute of Life Sciences, Scuola Superiore Sant'Anna, Pisa, Italy.}

Correspondence: Flavio Coceani (coceani@sssup.it)

\section{REFERENCES}

1. Goyal R, Goyal D, Longo LD, Clyman RI. Microarray gene expression analysis in ovine ductus arteriosus during fetal development and birth transition. Pediatr Res 2016;80:610-8.

2. Kajimoto H, Hashimoto K, Bonnet SN, et al. Oxygen activates the Rho/ Rho-kinase pathway and induces RhoB and ROCK-1 expression in human and rabbit ductus arteriosus by increasing mitochondria-derived reactive oxygen species. A newly recognized mechanism for sustaining ductal constriction. Circulation 2007;115:1777-88.

3. Costa M, Barogi S, Socci ND, et al. Gene expression in ductus arteriosus and aorta: comparison of birth and oxygen effects. Physiol Genomics 2006;25:250-62. 